\title{
化学健康篇
} 甲醛

刘哲, 冯天富, 陈建成 ${ }^{*}$

南京大学化学化工学院, 南京 210046

摘要: 讲述了主人公小泉造访新装修了房子的姑姑和姑父这一事件, 在这一事件中, 用主人公与姑姑和姑父的对话 来讲述甲醛对人体带来的危害、净化甲醛的方法以及其中蕴含的机理，还谈及甲醛的实际应用以及简要的原理。

关键词: 甲醛; 致癌; 甲醛净化; 聚甲醛塑料

中图分类号: G64; O6

\section{The Chemistry for Health: Formaldehyde}

\section{Zhe Liu, Tianfu Feng, Kin Shing Chan *}

School of Chemistry and Chemical Engineering, Nanjing University, Nanjing 210046, China.

Abstract: Through a story that the main character Xiaoquan visits the new house of his aunt and uncle, the series of dialogues on formaldehyde discloses the toxicity, the removal in indoor air and the chemistry mechanism. This article also introduced some practical uses of formaldehyde.

Key Words: Formaldehyde; Carcinogenicity; Formaldehyde removal; Polyformaldehyde plastics

\section{1 甲醛的危害}

小泉是一名大二化学专业非常优秀的本科生, 春节回家过年时, 姑姑家买了新房, 并且已经装 修完毕，便热情地邀请小泉到她家里做客，小泉爽快地答应了。

到达姑姑家门口，小泉按下门铃，很快姑姑和姑父便开门招呼小泉进屋。

刚一进屋, 小泉便闻到了一种刺鼻的气味, 小泉惊出一身冷汗, 这种气味正是甲醛的气味。小 泉赶忙询问姑姑: “姑姑，这房子装修好多长时间了？”

“刚过一周呢, 这不, 姑姑赶紧就请你来做客了, 今天还专门烧了你最爱吃的牛肉呢!”

“姑姑啊, 这房子装修完, 一般要等两三个月之后才能搬进来, 你们怎么不等等呢? ”

“还有这事吗, 我听你大婶她们说不碍事的呀！为什么一定要等两三个月才能搬进来呢？”

“姑姑, 你听我解释, 房子装修时用的油漆里面含有甲醛。你闻闻, 现在屋里是不是有一股怪味 儿?”

姑姑仔细嗅了嗅, 恍然大悟, 说道: “还真是有股味道, 但这个闻着似乎也没什么大问题, 你看 我和你姑父都住进来一周了也没什么事啊。”

“这是因为甲醛中毒是慢性中毒, 姑姑啊, 今天咱们就到外面吃饭吧, 这屋里实在是有害健康。

收稿: 2021-06-03; 录用: 2021-07-14; 网络发表: 2021-08-09

“通讯作者, Email: kschan@nju.edu.cn

基金资助：南京大学百位名师邀约项目; 南京大学国际化课程建设项目 
路上我也给你们仔细讲讲甲醛的危害, 你们可千万要听信我啊。”

“好, 姑姑相信你, 你本就是化学专业的, 姑姑不相信你相信谁啊? ” 姑姑笑着说, 并且招呼姑 父出门了。

姑父开车朝着饭馆出发, 在路上, 姑父刚一启动汽车, 便问: “小泉啊, 你懂得多, 你给姑父和 姑姑讲讲，那个什么醛到底有什么危害啊？”姑姑也随声附和。

“这甲醛啊, 对身体健康有很大影响。当室内浓度达到 $0.45 \mathrm{mg} \cdot \mathrm{m}^{-3}$ 时, 就会引起流泪以及眼睛异 常敏感等症状, 长期接触低剂量甲醛可引起慢性呼吸道疾病, 引起鼻咽癌、结肠癌、脑瘤等癌症。这 都是因为甲醛对皮肤粘膜有刺激作用, 长期暴露在有甲醛的环境中, 会造成咽喉、眼睛和鼻腔等部 位水肿、发炎、溃烂最终可能导致癌症。不仅如此, 一些人接触过甲醛还可能出现过敏现象, 严重 的甚至可能导致肝炎、肺炎及肝脏损害 ${ }^{[1]}$ 。总之甲醛是一种相当危险的化学物质, 可以的话最好不 要在浓度偏高的环境中久留。”

“啊, 居然这么严重!”姑姑和姑父大叫出来, 脸色都变了。

“不过不用担心，姑姑你们只需要暂时搬出来，等一段时间甲醛挥发完全再住进去就没事了。”

“小泉真是有见识, 等小宝宝以后出生了, 我定要叫他好好向你学习!” 姑姑摸着肚子高兴地说。

小泉又惊出一身冷汗, 虽然他也为姑姑有喜感到高兴, 但却无法直率地笑出来, 他紧张地补充 道: “姑姑啊, 甲醛可是会伤害你肚子里的小宝宝啊! 有研究表明, 甲醛可能会导致怀孕期间胎儿停 止生长发育, 心脑发育不全, 严重可致畸形和流产等严重后果, 还会增加自然流产的风险 ${ }^{[2]}$ ! 目前甲 醛已被世卫组织列为一级致癌和致畸形物质, 也就是明确了有致癌作用的物质! [3]”

姑姑的脸色铁青, 有些说不出话来。

“你先别急, 昨天咱才去做了胎检, 一切都正常, 应该不会有事的。” 姑父安慰着姑姑说道, 但 看得出他的脸色也不好看了。

“小泉啊, 真是太感谢你了, 要是真出了什么事, 我和你姑姑..... ”

“是啊，姑姑有你这个侄子真是太好了!”姑姑紧紧握着小泉的手，小泉也报以微笑，并且说道： “姑姑啊, 不用担心, 只要暂时搬出去就没问题了, 小宝宝会健康成长的, 等以后我可就能当哥哥了!” “哈哈, 好, 以后满月酒, 姑姑第一个请你!”

车里逐渐又充满三个人的欢声笑语, 小泉很庆幸自己学了化学, 能够在生活中保护身边的人。

\section{2 甲醛的净化}

三人在饭店吃完饭后, 小泉盛情邀请姑姑和姑父到自己家里先住一段时间, 姑父有些为难地说 道: “如果只住几天也就算了，但是要住那么长的时间，会给你们添麻烦的啊？”

“姑父不必担心, 我有几个妙招来快速除去甲酫, 这样你们就能很快搬进新家去了。”

“不愧是小泉, 真棒! 赶紧说给姑父听听呀。”

“好, 姑父你别着急, 听我细细讲来。首先姑父需要把家里的窗户全都打开, 这样有利于通风, 就能更好地将甲醛排出到室外。”

“好, 小泉, 别急, 等姑父记录一下。” 说罢, 姑父就从口袋里掏出手机, 打开记事本开始记录。

小泉见姑父记完一条, 便又接着说: “接下来啊, 姑父可以买些活性炭回来, 放在家里不通风的 地方。”

“什么炭？”姑父有些疑惑地问道, “炭原来还有这样的功效吗？”

“对啊,”姑姑也有些不解地问道, “咱们以前在农村也烧过炭, 那东西只会弄得煤灰到处都是, 怎么还能净化室内空气呢? 小泉, 你可得好好给我俩解释解释。”

“好, 这活性炭啊, 和以前咱们烧的普通的碳可不一样, 活性炭表面有很多肉眼看不见的小孔， 它能将甲醛这类有害的气体吸到那些小孔里并且牢牢地吸附住, 就像是海绵有很多小孔, 可以用来 吸水一样。” 
“哦, 我明白了。” 姑姑和姑父露出恍然大悟的表情, 姑父迅速将这一条记录在手机上。

“接下来啊, 还可以买一些甲醛清除剂, 定期喷一喷, 可以更快地消除甲醛。”

“这个甲醛清除剂又是什么原理啊, 能不能给我们讲讲? ”姑父好奇地问道。

“当然可以了, 市面上卖的甲醛消除剂中含有活性氨基, 它去除甲醛的方法就要涉及到我们化学 上的亲核加成了。”

“那个什么加成是怎么回事？”姑父的眼神中充满了好奇, 就连姑姑也被吸引了。

“这就相当于——小泉从书包里拿出两个条形磁铁, “你们看, 如果把这个条形磁铁代表甲醛, 甲醛最核心的部位就是羰基——碳和氧通过双键形成的基团, 氧上带有两对孤对电子, 而碳并不带 有孤电子, 但是碳周围的电子会被氧吸引过去, 这使得羰基具有很强的极性一一即氧带部分负电荷 而碳带部分正电荷。就像是这根磁铁一样, 我们让 $\mathrm{N}$ 极代表氧, $\mathrm{S}$ 极代表碳。而我们的甲醛清除剂中 的活性氨基的氮和氧一样带有孤对电子, 也相当于 $\mathrm{N}$ 极, ”小泉挥了挥手中的另一根磁铁, 话还没说 完就用手中磁铁的 $\mathrm{N}$ 极靠近桌上那一根磁铁的 $\mathrm{S}$ 极, 两者一下就结合在了一起, “ $\mathrm{N}$ 极和 $\mathrm{S}$ 极吸在一起, 即氨基的氮和羰基的碳相结合, 这样, 甲醛就被固定在了甲醛去除剂的液体中, 这就使得甲醛从空 气中被除去(图1)。”

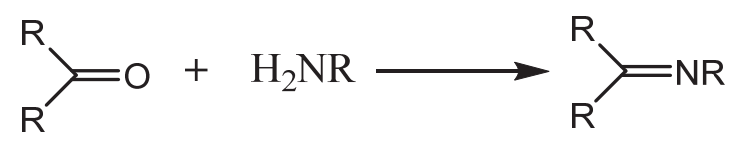

图1 甲酫与活性氨基的反应

“哦, 原来是这样啊!”姑父迅速地记录在手机中。

“小泉啊, 我之前看广告说过植物可以去除甲醛, 咱也可以买几盆盆栽回去养养吧? ”姑姑问道。

“当然可以养, 但是盆栽去除甲醛的能力并没有那么强。香港浸会大学在2010年发表的报告发现 植物降解甲醛的效率并不高, 每平方米的叶片每小时能降解的甲醛的量只有 $0.1 \mathrm{mg}$ 左右 ${ }^{[4]}$ 。”

姑父又问道: “那能不能用洋苾、风梨什么的来除去, 网上说用这些之后就没有甲醛的味道了? ”

“绝对不可以！这些植物只是掩盖了甲酫的气味，但并没有真正地去除甲醛。”

“原来是这样啊。”

“是啊, 只要做到我之前讲的几点绝对足够了!”

三人高高兴兴地离开饭店, 向小泉家出发了。

\section{3 甲醛的应用}

在车上, 姑父感叹道: “这个世界上要是少一点像甲醛这样的有毒物质, 咱们的生活就能更加健 康了啊。”

听完这话, 小泉笑着说: “那可不一定啊。”

姑父疑惑地问道: “此话怎讲?

小泉笑着说: “凡事都有两面性, 甲醛虽然有危害, 但却也有着各种各样的功能。比如聚甲醛塑 料, 这聚甲醛塑料还广泛用于汽车和消费电子产业, 其具有良好的耐磨性、耐热性、高刚性、低摩 擦等特点, 俗称塑钢、超钢, 在电器、电子、生活等应用领域都有聚甲醛塑料的身影。明明甲醛具 有毒性, 但却能广泛存在于我们的生活中, 真是天生我材必有用啊!” 小泉摸了摸汽车的门把手, 微 笑着看了看姑姑。

在一旁的姑姑坐不住了, 赶忙问道: “为什么甲醛这样的气体可以变成性能如此优秀的材料呢？” 小泉解释道: “这就是化学的厉害之处了! 首先让甲醛与水在酸性条件下反应, 此时水中的氧会 
对甲醛的羰基碳进行亲核进攻, 这样就会发生亲核加成, 得到水合甲醛, 再通过一系列反应得到甲 缩醛, 再加热甲缩醛, 此时甲醛就会被释放出来, 在催化剂的作用下逐渐聚合最终得到聚甲醛(图2)。”

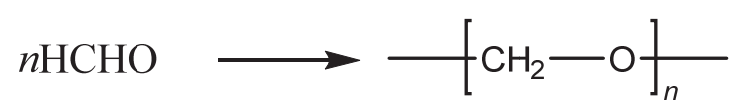

图2 甲醛聚合

“这个亲核进攻就是你刚才讲过的正负相吸, 没想到这还颇有一番中国传统阴阳学的智慧在其 中啊, 我现在算是听明白了, 最后甲醛聚合在一起也是因为这个什么亲核进攻吧, 之前你说过羰基 碳带部分正电, 氧带部分负电, 正好大量甲醛就可以通过碳和氧不断首尾连接下去, 这样就聚合成 我们肉眼可见的塑料了!”姑父激动地说道。

“的确是这样呀!”姑姑也想明白了，冲小泉坚起大拇指。

小泉又补充道: “姑姑、姑父啊, 你们应该听说过福尔马林吧？”

两人点点头, 表示知道, 姑父饶有兴致地问道: “难道福尔马林也是由甲醛通过亲核进攻来制备 的吗?”

小泉回答道: “这个就没有那么复杂了, 福尔马林通常是甲醛含量为 $37 \%$ 的水溶液, 在保存生物 体和防腐等方面应用广泛。不仅如此, 甲醛的水溶液还有杀菌消毒的功能, 特别是在类毒素疫苗的 生产过程中, 往往就是使用甲醛来灭活相应的细菌, 还可以杀死不需要的病毒和病菌以防污染疫苗。”

“姑姑倒是知道防腐，但为什么甲醛可以消毒杀菌呢？”姑姑和姑父又有些疑惑起来。

“这个啊, 且听我细细讲来!”

小泉清了清嗓子, 继续说道: “这就要从蛋白质说起了, 首先蛋白质的种类数不胜数, 它们有各 种各样的功能来维持我们生命体的正常运作, 不管是人类、小动物, 还是昆虫、细菌, 要正常地生 存下去就需要结构和功能完整正常的蛋白质, 否则最终就会危害生命体的健康, 甚至导致生命体死 亡。”

姑姑想了想问道: “也就是说甲醛可以破坏细菌的蛋白质从而做到杀菌消毒吗？”

“不愧是我的姑姑，你说对了!”小泉坚起大拇指夸奖道。

“哪里, 都是小泉讲得好, 连姑姑都能听明白。”

“那甲醛是如何破坏细菌的蛋白质的呢？”姑父的语气中充满着好奇。

“这就要谈一谈蛋白质的结构了, ” 小泉不紧不慢地说道, “首先蛋白质是由氨基酸组成的, 而组 成蛋白质的氨基酸都有一个共同的特点, 就是具有氨基。之前我们也知道了, 利用甲醛去除剂除尽 室内甲醛时就利用了活性氨基来亲核进攻甲醛的羰基碳, 这里的蛋白质与甲醛作用的方式也是类似 的。”

“哦, 我明白了, 刚才你说蛋白质由氨基酸组成, 而这些氨基酸上都具有这样的氮原子, 那这 些氮原子就能亲核进攻甲醛从而与甲醛相连!”姑姑激动地说出了自己的想法。

“没错, 与大量的甲醛相连会导致蛋白质本身的结构遭到破坏(图3), 最终甚至还可能会形成沉 淀, 但总的结果就是蛋白质的结构被破坏失去功能, 这样, 病菌也好, 病毒也罢, 都难以维系自身 的生存了, 也就达到了杀菌消毒的效果。”

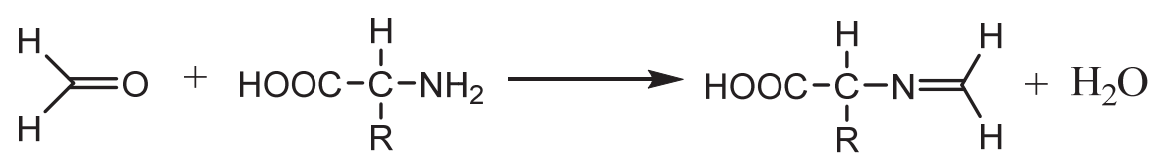

图3 氨基酸与甲酫的结合 


\section{4 结语}

姑姑和姑父到了小泉家, 向小泉的父母夸奖了小泉, 并且感叹道, 这甲醛真是奇妙, 虽然会危 害人的健康, 但是也能杀灭细菌, 制作各种材料, 为我们的出行安全保驾护航, 真可谓是健康的标 尺啊！

\section{考 文 献}

[1] 翟淑妙, 徐晓俨, 张九乾. 环境与健康杂志, 1994, 11 (5), 238.

[2] 陈晨, 程义斌. 卫生研究, 2015, 2 (44), 312.

[3] 世界卫生组织国际癌症研究机构致癌物清单. [2021-04-28]. https://www.nmpa.gov.cn/xxgk/mtbd/20171030163101383.html

[4] 植俊宁, 余振辉, 吴志辉, 李朝康, 阮华达. 中国农学通报, 2010, 26 (2), 196. 